As a UK family doctor, I was particularly struck by how seldom Tobago's Health Centre patients 'medicalised' emotional distress, in contrast to my UK experience, where 'minor' psychological disorders are a significant part of every GP's daily work.

S. P. Cembrowicz

Montpelier Health Centre, Bristol BS6 5PT

\section{Detention under Section 3 of the Men- tal Health Act and home security}

Sir: When a patient is admitted to hospital under Section 3 of the Mental Health Act, the approved social worker is required to ensure that their residence is made secure. However, once this is done there is no requirement to continue to maintain security at the site. We have recently had experience of a patient whose entire belongings were stolen while receiving treatment as an in-patient. This was not only extremely distressing to the patient, but also delayed rehabilitation and discharge. Discussion with colleagues suggests that this is not an uncommon experience.

Patients detained under Section 3 usually have chronic psychotic illnesses and frequently live alone in housing that is less than ideal. They may be well-known in the neighbourhood and prolonged absence is clearly noted and acted on. As their detention is at the instigation of the psychiatric services, we feel that those services should carry some responsibility for maintaining the security of our patients' property. Especially as we usually justify compulsory detention as being in their interest.

We suggest that the team involved in the care instigate arrangements for regular (weekly) visits to the home to ensure it remains secure. Perhaps it would even be appropriate to make this aspect of patient care and support statutory. It is unfortunate that sometimes this aspect of a patient's social care is not considered, especially as it is obviously very important to them.

RICHARD C. BARNES, JUDITH ORRELL and ROBERT BROWN

Rehabilitation and Special Care Directorate,

Rathbone Hospital, Mill Lane, Liverpool L9 7JP

\section{Catchment areas}

Sir: Kellett (Psychiatric Bulletin, June 1995, 19. 240-342) and Thornicroft et al (Psychiatric Bulletin. June 1995. 19 343-345) present arguments for and against the geographical catchment area. No system is perfect but having been a consultant operating within and without the catchment area I have to vote in favour. I think Kellett is wrong in saying there are no longer valid reasons for a catchment area, particularly when his own perceived benefits of the system seem to encompass many of the fundamentals of good psychiatric practice while his list of 'harms' contains little to do with patients.

At the request of our purchasers we have transferred from geographical catchment areas to consultants being linked to named general practitioners (GPs) who are grouped to produce 'neighbourhoods'. This was introduced as a purchasing strategy to allow groups of GPs (neighbourhoods) working in similar areas and experiencing similar problems to identify local service need in their dialogue with purchasers and providers. But these problems are very strongly geographically linked and this is demonstrated by our annual public health reports. The majority of GPs' patients reside in a local area but GPs are not geographically confined and can have patients widely dispersed. They tell me they have to keep patients living further away to maintain their list size and stay solvent. Our neighbourhood arrangement means the consultant seeing the patient is determined by the GP's name though the GP has a choice of two consultants. Consequently, consultant patients are now spread over a larger geographic area than before.

While working with geographical areas I was able to establish community out-patient clinics where patients are reviewed in their own home. This system will only work if the population served generates a manageable caseload but more importantly is sufficiently concentrated in a geographical area to minimise time lost travelling between houses. Now that I track GPs I have to travel further, the number of patients I can see in a session will inevitably drop and the cost of the clinic will rise. The clinics may become nonviable.

The community clinic is exceptionally popular with patients, does away with tedious ambulance arrangements, dramatically reduces non-attendance and meets the needs of elderly people with high levels of physical and mental disability in the inner city who cannot easily use traditional services. Would it be progress to abandon a development of this sort?

I still believe the geographical catchment area provides a good basis for the delivery of mental health services. It facilitates the identification of local needs, close liaison between disciplines and the development of service and expertise relevant to a locality. Kellett is quite wrong in suggesting the purpose of locality-based services is to take over complete care of the patient. On the contrary, detailed knowledge of the locality 
enables me as a psychiatrist to withdraw from treatment knowing the competency of support systems that will not alert services to future problems. Kellett's view that psychiatric services have moved away from the care of psychotic patients to allow more work with neurotic disorders in the community is not my experience of inner city psychiatry where our time is increasingly dominated by the care of serious mental illness.

Many of the problems in the for or against debate could be solved if the organisation of general practice were to change. If GPs become locality-based there could be co-terminous relationships between GP and specialist based on geographical catchment areas. GPs work the way they do for financial not clinical reasons. Choice of GP does not really exist for disabled, vulnerable patients who are practically bound to the local general practice.

Finally. Kellett suggests that mediocrity results from catchment areas stifling competition between consultants. Consultants are well paid professionals who have a contracted responsibility to develop and deliver a quality service with or without competition. Mediocrity is the result of mediocre doctors who believe they can make the minimum of effort once in a permanent position knowing there is lack of sanction against those who do a poor job. Ultimately, if all doctors were good at what they did there wouldn't be a problem. I doubt we can blame the catchment areas for that one.

DAVID N. ANDERSON

Department of Psychogeriatrics,

EMI Directorate, Sir Douglas Crawford Unit,

Mossley Hill Hospital, Park Avenue.

Liverpool L18 8BU

\section{Bias in the assessment of psychiatric emergencies}

Sir: We read with interest the article by Hall $\&$ Deahl on the inadequacies of history taking by trainee psychiatrists in casualty (Psychiatric Bulletin. September 1995, 19. 538-540). While we agree that efforts are merited to increase alcohol and substance abuse histories in all groups, we disagree that this discrepancy is likely to represent ageist or sexist attitudes. The OPCS survey (Goddard, 1991) of drinking habits in the late 1980s (quoted in part in Hall \& Deahl's article) found that $23 \%$ of men and $8 \%$ of women exceeded sensible drinking levels (21 units for men and 14 units for women). Excess drinking showed a decline with increasing age in both sexes. Based on these figures, if a full alcohol history had been taken in all cases at least a further $5.3 \%$ of men and $3.4 \%$ of women would have been identified as exceeding sensible drinking levels. The recording of disorders more likely to occur in a sub-population has a long history in medicine and we do not feel it should necessarily be dismissed as ageist or sexist.

\section{Reference}

GODDARD. E. (1991) Drinking in England and Wales in the late 1980s. London: HMSO/OPCS.

Michel Vanstraelen

Prudhoe Hospital, Prudhoe

RICHARD DUFFETT

Unit of Human Psychopharmocology.

The Royal London Hospital (St Clements), London

Sir: We agree with Vanstraelen \& Duffet that substance use occurs at different levels in different population subgroups. We also agree that it is clinically important to recognise such differences. However, in emergency clinic psychiatry we think that to let information about populations lead us into assumptions about individuals is clinically dangerous. One cannot exclude a diagnosis just because it is unlikely.

IAN HALL

St George's Hospital Medical School.

University of London

MaRTIN DEAHL

St Bartholomew's Hospital Medical College, London

\section{Masters courses in psychiatry}

Sir: We thought that the article by Shoebridge \& McCartney (Psychiatric Bulletin, September 1995. 19, 555-558) raised interesting questions. Having experienced the Cardiff MSc course, we would make the following points:

(1) The development of the MSc course acted as a catalyst to enhance greatly the quality of training for the MRCPsych in Cardiff. Few of us would see any advantage in a return to the 'old' MRCPsych course.

(2) In a rotation which is spread across South Wales, the MSc course has provided a focus and a route of access to the expertise of the academic department in Cardiff for supervision and advice.

(3) The research component means that trainees are encouraged to undertake research and learn research methodology earlier than they might.

(4) At its best, it provides a higher degree in psychiatry and a publication around the 